\title{
Estudo da variabilidade da radiação solar no Nordeste do Brasil
}

\author{
Roberta A. e Silva ${ }^{1}$, Vicente de P. R. da Silva', Enilson P. Cavalcanti ${ }^{1} \&$ David N. dos Santos ${ }^{1}$
}

\begin{abstract}
RESUMO
A finalidade deste trabalho é avaliar as tendências das séries temporais do saldo radiação no Nordeste do Brasil, através de dados de reanálise do NCEP/NCAR referentes ao período de 1948 a 2006. As séries temporais anuais de dados observados na superfície de radiação solar global e evaporação do tanque classe "A" de duas localidades do semiárido da região de estudo, também foram utilizadas. Analisou-se a variabilidade temporal das séries temporais com base no teste não-paramétrico, de Mann-Kendall. As séries temporais do saldo de radiação exibiram reduções acentuadas entre 1948 e 1987, que foram estatisticamente significativas em níveis de 0,01 de probabilidade pelo teste de Mann-Kendall; verificou-se, entretanto, no período de 1988 a 2006, comportamento inverso, predominando tendências positivas de saldo de radiação. $0 \mathrm{~s}$ resultados também indicaram que os dados observados de radiação global e de evaporação do tanque classe "A", apresentam reduções acentuadas ao longo do período estudado estatisticamente significativos em níveis de 0,01 de probabilidade. Esses resultados sugerem a presença do fenômeno "Global dimming" sobre a região Nordeste do Brasil.
\end{abstract}

Palavraschave: evaporação do tanque classe "A", global dimming, teste de Mann-Kendall

\section{Study of the variability of solar radiation in northeast Brazil}

\begin{abstract}
The main objective of this work was to assess the tendency of time series of net radiation in the northeastern region of Brazil from the NCEP/NCAR database of the 1948-2006 period. Also, the annual time series of data observed at surface of global solar radiation and class A pan evaporation for two stations located in the semiarid area of studied region were analyzed. Long-term variability of the time series was assessed by the M ann-Kendall test. The time series of net radiation showed a reduction through the 1948-1987 period, statistically significant at the 0.01 level of probability by the MannKendall test. However, the 1988 to 2006 period showed an inverse pattern in net radiation with positive trends. Results also indicate that observed data in global solar radiation and class A pan evaporation at Petrolina, PE and Juazeiro, BA presented an accentuated decrease through the study period statistically significant at 0.01 level of probality by the MannKendall test. These results suggest that the global dimming effect is present in the northeastern region of Brazil.
\end{abstract}

Key words class A pan evaporation, global dimming, Mann-Kendall test

1 UACA/UFCG, Av. Aprígio Veloso, 882, Bodocongó, CEP 58109-970, Campina Grande, Paraíba. Fone: (83) 3310-1202; 3310-1201. E-mail: beta_araj@yahoo.com.br; vicente@dca.ufcg.edu.br; enilson@dca.ufcg.edu.br; david_nsantos@yahoo.com.br 


\section{INTRODUÇÃO}

A radiação solar é a principal fonte de energia para os processos físicos, químicos e biológicos que ocorrem no sistema Terra-atmosfera. O conhecimento sobre esta variável acerca da sua variabilidade, tem aplicabilidade em diversas áreas científicas, como engenharia, arquitetura, meteorologia, agricultura, hidrologia e, ainda, como indicador da variabilidade climática (Lohmann et al., 2006). Nas últimas décadas diversas pesquisas apontam variações temporais na taxa de radiação solar. Uma das primeiras pesquisas em que se abordou este tema, foi aquela desenvolvida por Ohmura \& Lang (1989) que observaram que as taxas de radiação solar incidente à superfície terrestre estariam declinando de maneira acentuada, desde 1950. Anos mais tarde, os mesmos resultados encontrados por esses autores foram observados na antiga União Soviética (Russak, 1990; Zhitorchuk et al., 1994); na Austrália (Stanhill \& Kalma, 1994); na Alemanha (Liepert, 1994); em Hong Kong (Stanhill \& Kalma, 1995; Abakumova, 1996), em Israel (Stanhill \& Ianetz, 1996), na Antártica (Stanhill \& Cohen, 1997; Liepert, 1996) e no Brasil (Silva et al., 2008). Stanhill \& Cohen (2001) denominaram este decréscimo acentuado da radiação solar que chega à superfície da Terra tanto pelos raios solares diretos como pela radiação solar difusa, de "Global dimming”.

Liepert \& Tegen (2002) argumentam que o efeito "Global dimming” é devido ao aumento das partículas de aerossol na atmosfera. Nazarenko \& Menon (2005), ao observarem o comportamento da radiação solar em duas condições distintas com e sem a presença de aerossóis, utilizando um modelo acoplado a um modelo dinâmico do oceano, verificaram que, sem a presença de aerossóis, a radiação solar absorvida à superfície terrestre aumentou durante o período analisado (1960 a 2002). Outra provável causa do “Global dimming” seria a variabilidade na frequência de nebulosidade (Liang \& Xia, 2005; Qian et al., 2006). Os estudos mais recentes (Philipona et al., 2004; Pallé et al., 2004; Charlson et al., 2005) sugerem a ausência de "dimming” a partir da década de 90, em diversas partes do globo, principalmente na América do Norte, Europa Ocidental e China.

As consequências da variabilidade da radiação solar observadas nas últimas décadas, podem ser catastróficas para diversas atividades humanas, como destacaram Nazarenko \& Menon (2005). A inversão do "Global dimming” pode acarretar efeitos desastrosos sobre o clima, especificamente em relação ao ciclo hidrológico, na temperatura da superfície da Terra e na precipitação pluvial. Estudos revelam que a evaporação decresceu sobre a China (Thomas, 2000), Austrália (Roderick \& Farquhar, 2004) e na Índia (Tebakari et al., 2005). Por outro lado, tendências positivas desta variável foram observadas em Israel por Cohen et al. (2002). Todas essas pesquisas se concentram no Hemisfério Norte e/ou nas regiões de intensa atividade industrial. Neste contexto se pretende, no presente trabalho, oferecer uma contribuição positiva visando a um conhecimento melhor do efeito "global dimming” na região Nordeste do Brasil, região que apresenta pequena concentração industrial, com $60 \%$ de sua área total na parte semi-árida, com duração do dia solar médio de aproximadamente $12 \mathrm{~h}$ registrando fluxos instantâneos de até $1200 \mathrm{~W} \mathrm{~m}^{-2}$, ou seja, dispõe de condições climáticas favoráveis à utilização da energia solar como fonte primária de energia a ser transformada em energia elétrica.

A importância da utilização da energia solar como fonte de energia renovável ou, ainda, em outras atividades humanas, requer um conhecimento maior acerca da sua variabilidade porém informações confiáveis sobre essa variável são raras. Investigações referentes à radiação solar, enfrentam muitas limitações em função da baixa densidade de estações radiométricas sobre a região Nordeste do Brasil; esta situação tem incentivado alguns pesquisadores a adotarem modelos baseados em dados de satélites (Ceballos \& Bottino, 2002; 2004), a fim de suprimir a baixa densidade de estações actinógrafas sobre o Brasil.

Os dados de reanálise do NCEP/NCAR, ainda constituem uma ferramenta pouco difundida para essas análises, embora apresentem uma série temporal consistente de dados históricos da atmosfera, permitindo a obtenção da radiação solar sobre regiões extensas e isoladas onde não seria possível instalar estações actinógrafas e ainda com boa variabilidade temporal (desde 1948 até 2009); desta forma, referidos dados oferecem um potencial de medida de fluxos radiativos sobre grandes áreas e sobre regiões em que não existe uma densa rede de estações, resultando em alternativa para o monitoramento da radiação solar à superfície com boa variabilidade espacial e temporal.

Assim e se considerando a grande importância do tema aqui abordado, é que se propôs, neste trabalho, analisar prováveis tendências nas séries temporais de saldo radiação do NCEP/NCAR e também, de dados de radiação solar global e de evaporação do tanque classe "A" observados à superfície no período de 1975 a 2006, para avaliar a possível presença do efeito "global dimming” no Nordeste brasileiro.

\section{MATERIAL E MÉTODOS}

\section{Área de estudo}

A região de estudo compreende a região Nordeste do Brasil (NEB) situada ao Sul do Equador, entre $1^{\circ}$ e $18^{\circ} 30^{\prime}$ de latitude Sul e $34^{\circ} 20^{\prime}$ e $48^{\circ} 30^{\prime}$ de longitude Oeste tendo, a Norte e a Leste, o Oceano Atlântico. O NEB é conhecido pela predominância de características climáticas semiáridas e pelas altas variabilidades temporal e espacial da precipitação pluvial, com grande impacto socioeconômico sobre a região (Silva et al., 2006). A população que habita esta região sofre frequentemente as consequências da instabilidade climática, tal como a deficiência no suplemento de água que abastece a região e perdas agrícolas. Esta extensa área é habitada por mais de 30 milhões de pessoas e a economia se baseia, essencialmente, na agricultura de subsistência (Silva, 2004).

\section{Dados utilizados no estudo}

Com vistas à realização deste trabalho, utilizaram-se dados mensais de saldo de radiação da área correspondente ao Nordeste Brasileiro, para o período de 1948 a 2006, obtidos 
na base de dados do National Center for Environmental Prediction/National Center for Atmospheric Research (NCEP/ NCAR), com resolução de $2,5^{\circ} \times 2,5^{\circ}$ graus de latitude e longitude. As séries temporais utilizadas neste estudo foram submetidas a análise de consistência de dados. A Figura 1 exibe a distribuição espacial dos pontos de grades da região Nordeste do Brasil, analisados neste estudo.

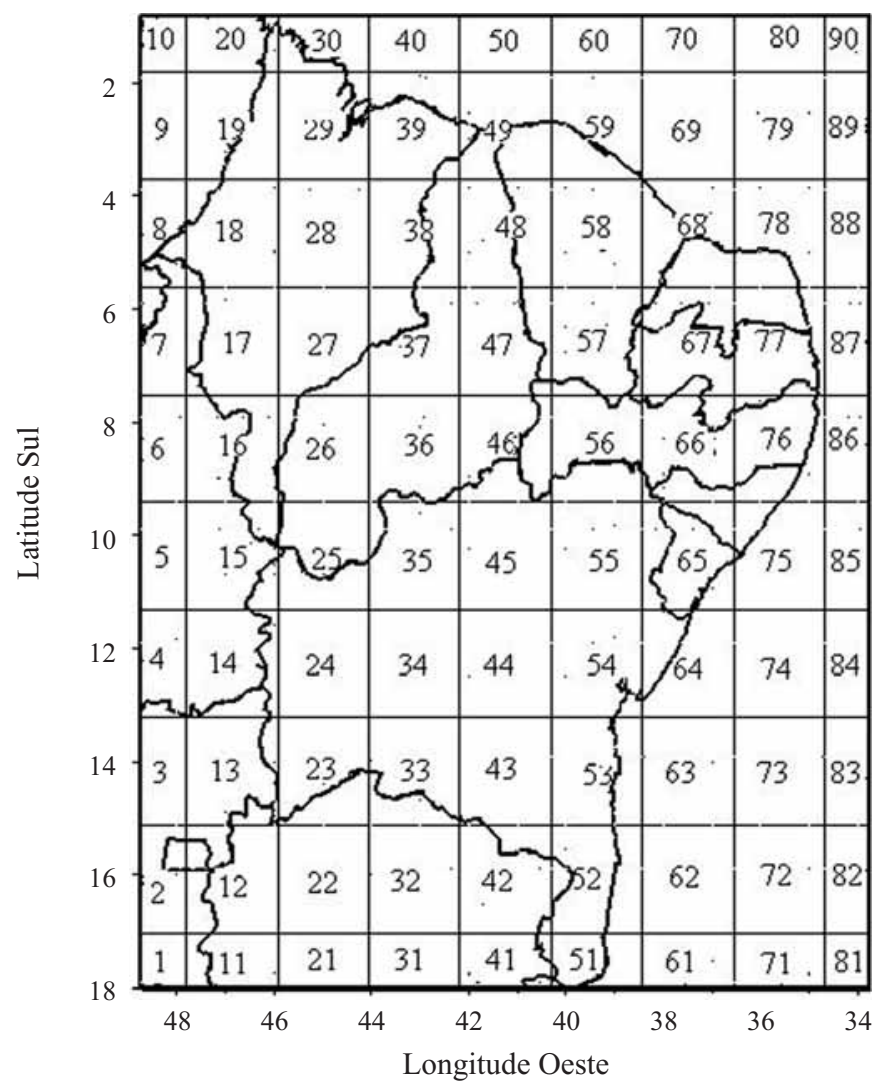

Figura 1. Distribuiccão espacial dos 90 pontos de grade, com resolução de $2,5^{\circ} \times 2,5^{\circ}$ graus de latitude e longitude, sobre a região $\mathrm{N}$ ordeste do Brasil

Analisaram-se, também, as séries temporais de radiação solar global e de evaporação do tanque classe "A" de duas localidades do semiárido da região de estudo: Juazeiro, BA (latitude $09^{\circ} 24^{\prime} \mathrm{S}$ e longitude $40^{\circ} 26^{\prime} \mathrm{W}$ ) e Petrolina, PE (latitude $09^{\circ} 09^{\prime} \mathrm{S}$ e longitude $40^{\circ} 22^{\prime} \mathrm{W}$ ), no período de 1975 a 2006. Obtiveram-se os dados dessas localidades no banco de dados meteorológicos da Embrapa Semi-Árido.

Nesta pesquisa se determinaram as tendências temporais (por década e por período de estudo) das séries temporais do saldo de radiação, derivadas dos dados de reanálise do NCEP/ NCAR os quais, por sua vez, foram obtidos no formato de arquivos binários, oriundos da web site: www.dca.noaa.gov/ cdc/data.ncep.reanalysis.derived, posteriormente convertidos em arquivos txt, para serem visualizados em planilha Excel. Médias anuais para cada ponto da grade dos 90 pontos sobre a região Nordeste do Brasil, foram geradas; em seguida, utilizando-se os valores médios anuais dessa variável, determinaram-se as tendências temporais e os níveis de significância estatística correspondentes a cada ponto de grade. As tendências e suas respectivas significâncias foram definidas, inicialmente, por década e, posteriormente, para o período de estudo. Para a espacialização das tendências e dos níveis de significância das séries temporais, utilizou-se o software Grid Analysis and Display System (GrADS).

\section{Análise de tendência}

A análise da tendência das séries temporais foi realizada através do teste de Mann-Kendall (Mann, 1945; Kendall, 1975). Este teste não paramétrico é sugerido pela Organização Meteorológica Mundial (OMM) para avaliação da tendência em séries temporais de dados ambientais (Yue et al., 2002). Para Modares \& Silva (2007) o teste de Mann-Kendall avalia com bastante eficiência a tendência de séries temporais de dados ambientais; trata-se de um teste que compara cada valor de uma série temporal com os outros valores restantes, sempre em ordem sequencial (Silva et al., 2007). A estatística do teste é:

$$
\mathrm{S}=\sum_{\mathrm{i}=2}^{\mathrm{n}} \sum_{\mathrm{j}=1}^{\mathrm{i}-1} \sin \mathrm{al}\left(\mathrm{x}_{\mathrm{i}}-\mathrm{x}_{\mathrm{j}}\right)
$$

em que $x_{j}$ são os dados estimados da sequência de valores, $n$ e o número de elementos da série temporal e o sinal $\left(\mathrm{x}_{\mathrm{i}}-\mathrm{x}_{\mathrm{j}}\right)$ é igual a -1 para $\left(\mathrm{x}_{\mathrm{i}}-\mathrm{x}_{\mathrm{j}}\right)<0,0$ para $\left(\mathrm{x}_{\mathrm{i}}-\mathrm{x}_{\mathrm{j}}\right)=0$, e 1 para $\left(x_{i}-x_{j}\right)>0$. Kendall (1975) mostrou que $S$ é normalmente distribuída com média E(S) e variância $\operatorname{Var}(\mathrm{S})$, que para uma situação na qual pode haver valores iguais de $\mathrm{x}$, são calculadas pelas equações:

$$
\begin{aligned}
& \mathrm{E}[\mathrm{S}]=0 \\
& \operatorname{Var}[\mathrm{S}]=\frac{\mathrm{n}(\mathrm{n}-1)(2 \mathrm{n}+5)-\sum_{\mathrm{i}=1}^{\mathrm{q}} \mathrm{t}_{\mathrm{p}}\left(\mathrm{t}_{\mathrm{p}}-1\right)\left(2 \mathrm{t}_{\mathrm{p}}+5\right)}{18}
\end{aligned}
$$

em que $\left(\mathrm{t}_{\mathrm{p}}\right)$ é o número de dados com valores iguais em certo grupo (pth) e q é o número de grupos contendo valores iguais na série de dados em um grupo p; o segundo termo representa um ajuste para dados censurados.

Usando-se a Eq. 1, tem-se que o valor positivo de S indica tendência também positiva dos dados que crescem com o tempo; por outro lado, o valor negativo indica tendência decrescente. Sabendo-se que S é normalmente distribuída e que tem média zero e variância dadas pelas Eqs. 2 e 3, respectivamente, pode-se testar se a tendência positiva ou negativa é significativamente diferente de zero; se $\mathrm{S}$ é significativamente diferente de zero, a hipótese nula $\mathrm{H}_{0}$ pode ser rejeitada para certo nível de significância apontando para a existência de tendência; inversamente, a hipótese alternativa $\left(\mathrm{H}_{1}\right)$ é aceita. $\mathrm{O}$ teste estatístico parametrizado $\left(\mathrm{Z}_{\mathrm{MK}}\right)$ é computado pela seguinte equação:

$$
\mathrm{Z}_{\mathrm{MK}}= \begin{cases}\frac{\mathrm{S}-1}{\sqrt{\operatorname{Var}[\mathrm{S}]}} & \text { para } \mathrm{S}>0 \\ 0 & \text { para } \mathrm{S}=0 \\ \frac{\mathrm{S}+1}{\sqrt{\operatorname{Var}[\mathrm{S}]}} & \text { para } \mathrm{S}<0\end{cases}
$$


A presença de uma tendência estatisticamente significativa é avaliada usando-se o valor de Z; tal estatística é empregada para testar a hipótese nula, isto é, que nenhuma tendência existe. Um valor positivo de $\mathrm{Z}_{\mathrm{MK}}$ indica um aumento da tendência e, quando negativa, aponta tendência decrescente. Para testar a tendência monotônica crescente ou decrescente no nível de significância de $\mathrm{p}$, a hipótese nula será rejeitada se o valor absoluto de $\mathrm{Z}$ for maior que $\mathrm{Z}_{1-\mathrm{p} / 2}$, utilizando-se a tabela da distribuição normal cumulativa padrão. Os níveis de significância de p = 0,01 e 0,05 foram aplicados neste estudo.

\section{RESULTADOS E DISCUSSÃO}

Saldo de radiação nas décadas de (1948-1957) e de (1958-1967)

A distribuição espacial das tendências temporais do sal-

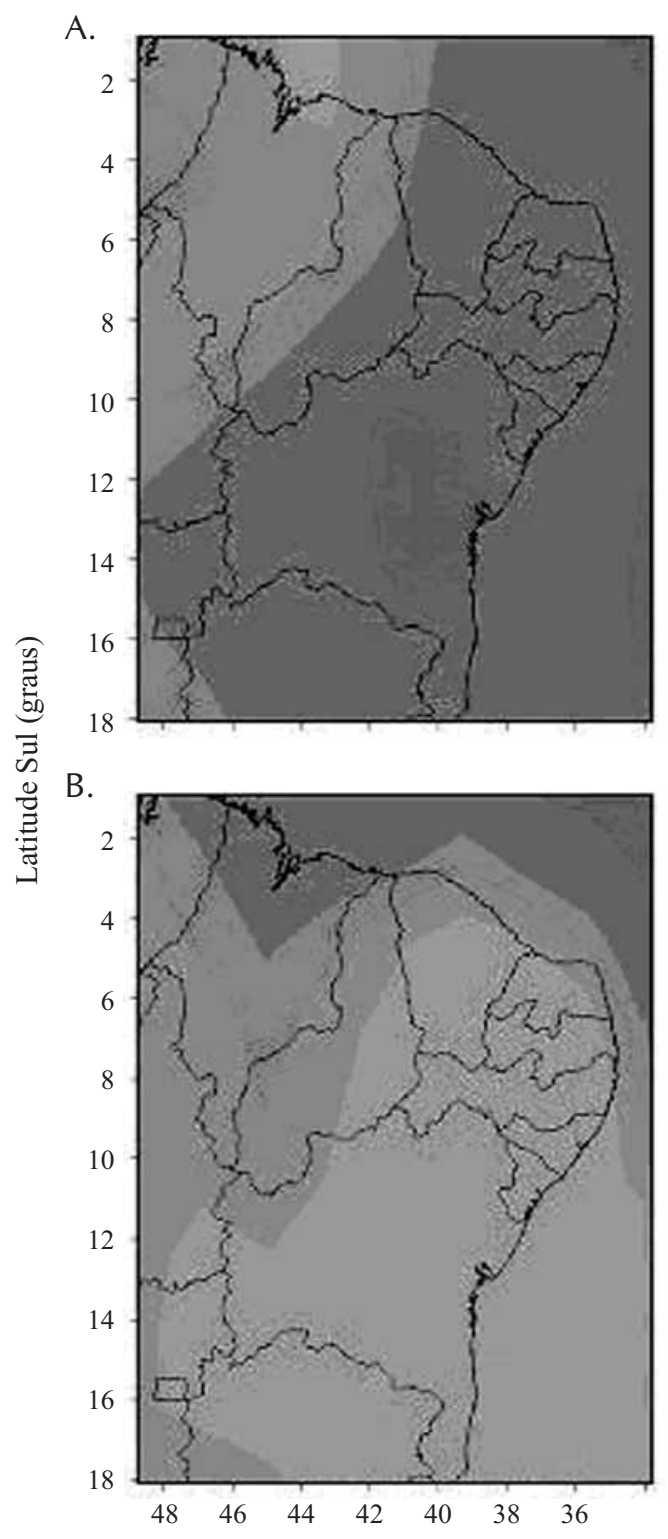

do de radiação obtidas com base nos dados do NCEP/NCAR, da primeira década do período de estudo (1948-1957) evidencia a ocorrência de um acréscimo desta variável sobre grande parte da região Nordeste do Brasil; entretanto se observam, sobre o Estado do Maranhão, tendências negativas (Figura 2A). Nota-se que apenas a parte centro-oeste do NEB apresenta tendências positivas estatisticamente significativas a níveis de 1 e 5\% de probabilidade (Figura 2C); por outro lado, na década 1958-1967 deu-se inversão no comportamento das tendências de saldo de radiação, com relação à década anterior (Figura 2B).

Em toda a área de estudo ocorreram ampliação das tendências negativas e diminuição das áreas com tendências positivas do saldo de radiação, com base nos dados do NCEP/ NCAR. Essas tendências no Nordeste do Brasil são estatisticamente significativas pelo teste de Mann-Kendall a níveis de significância de 1 e 5\% de probabilidade (Figura 2D).

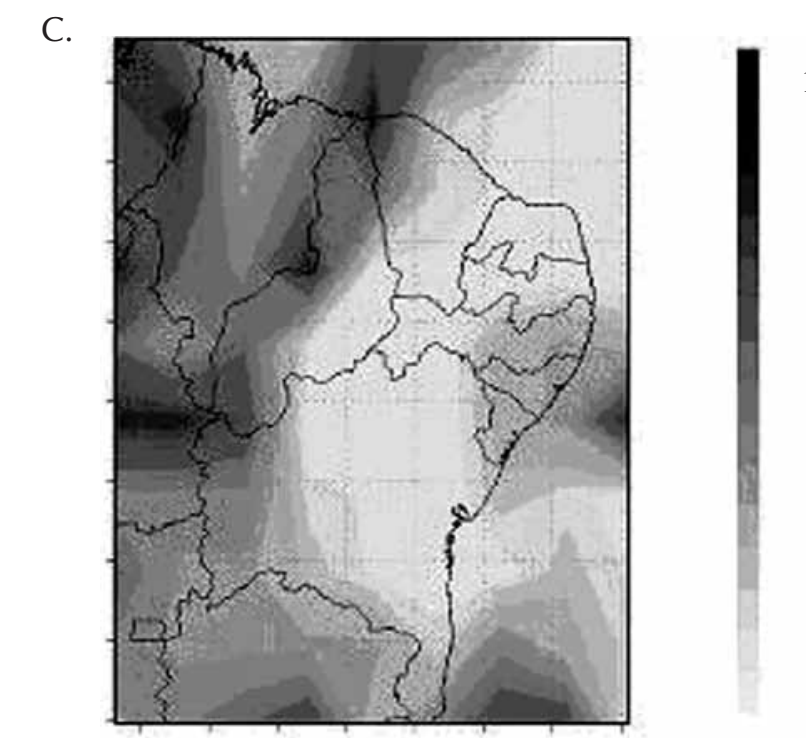

100

D.

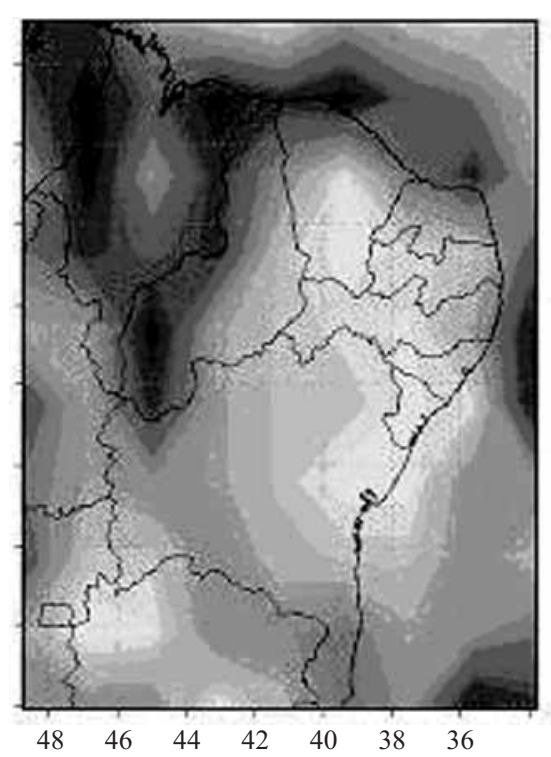

Figura 2. Distribuicão espacial das tendências do saldo de radiação das décadas de 1948-1957 (A) e de 1958-1967 (B) e distribuição espacial dos valores dos níveis de significância das tendências do saldo de radiação das décadas de 1948-1957 (C) e de 1958-1967 (D) no Nordeste do Brasil 
Saldo de radiação nas décadas de 1968-1977 e 1978-1987

O saldo de radiação das décadas 1968-1977 e 1978-1987, apresenta homogeneidades na distribuição das tendências (Figuras 3). Essas tendências foram predominantemente negativas sobre quase todo o NEB durante as referidas décadas, com exceção de uma faixa com valores positivos cobrindo o nordeste da Bahia, oeste de Pernambuco e o sul do Ceará, além de todo o Estado de Sergipe.

Verificam-se valores de tendência do saldo de radiação estatisticamente significativos a níveis de 1 e 5\% de probabilidade nas décadas 1968-1977 e 1978-1987, a oeste do NEB, e sobre uma estreita faixa a leste dos Estados do Rio Grande do Norte, Pernambuco e Paraíba (Figuras 3C e D).

Saldo de radiação nas décadas de 1988-1997 e 1998-2006 Nas duas últimas décadas do período estudado (19881997) e de (1998-2006), verificou-se mudança na distribui-

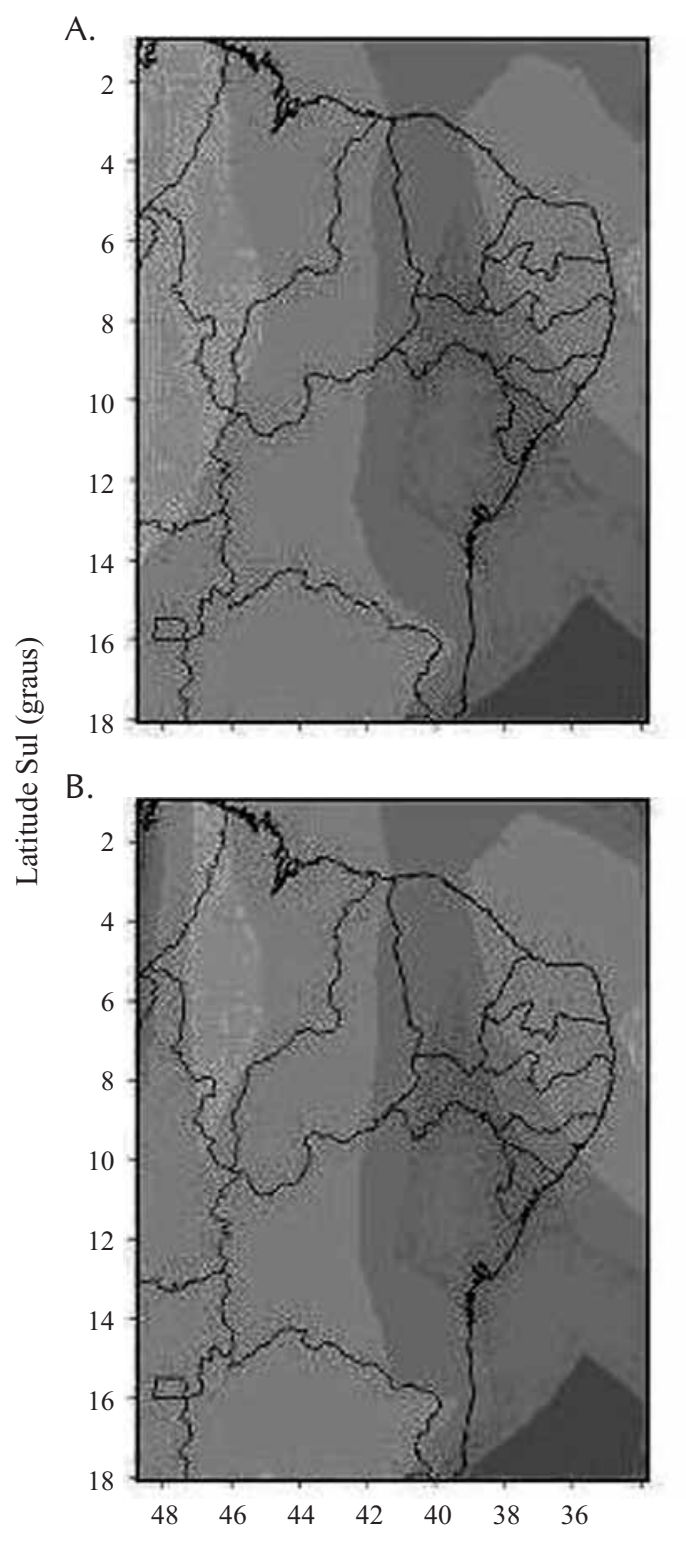

4,0

3,0

,0

3,0 ção espacial das séries temporais do saldo de radiação (Figuras 4A e B), predominando tendências positivas sobre quase toda a região de estudo.

Na quinta década (1988-1997), o saldo de radiação apresentou um núcleo com tendências negativas porém sem qualquer significância estatística (Figura 4C). Quanto à significância estatística da sexta década (1998-2006), observam-se núcleos de tendências positivas localizadas no noroeste da região de estudo, com nível de significância de 5\% de probabilidade (Figura 4D). Nesta década também se observam tendências negativas no sudoeste do Estado da Bahia mas sem qualquer significância estatística pelo teste de MannKendall.

Saldo de radiação no período total de estudo (1948-2006)

A distribuição espacial da tendência temporal do saldo de radiação e dos valores do nível de significância da tendên-
C.

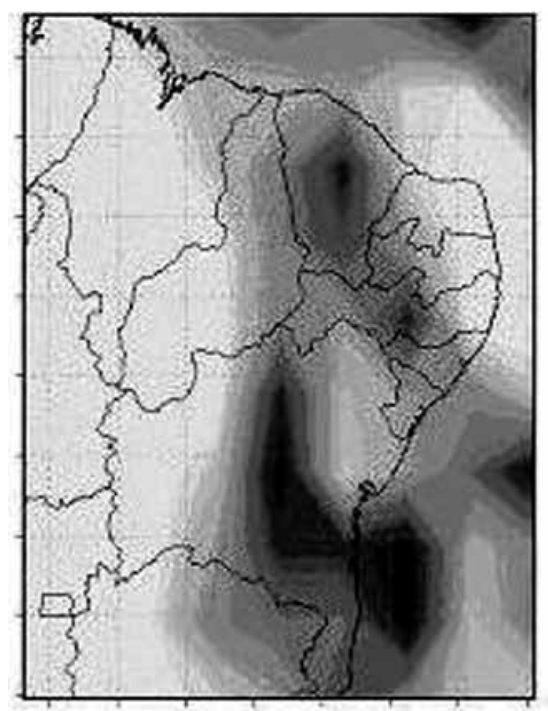

D.

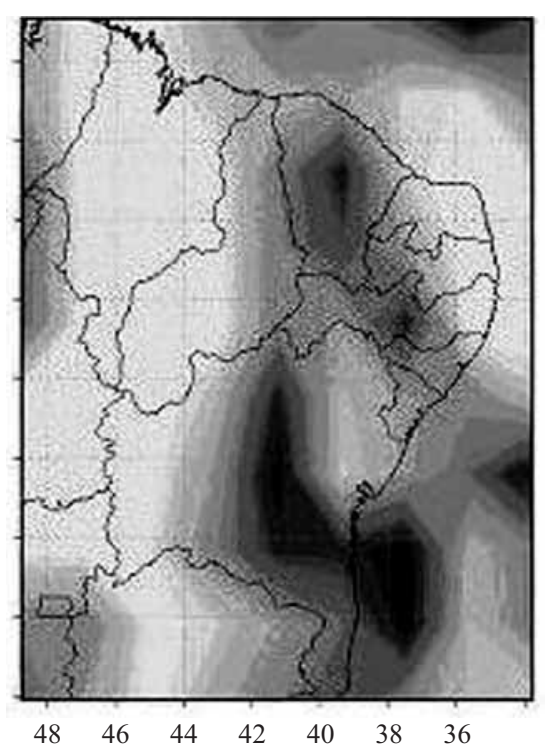

100

00

Figura 3. Distribuicão espacial das tendências do saldo de radiação das décadas de 1968-1977 (A) e de 1978-1987 (B) e distribuição espacial dos valores dos níveis de significância das tendências do saldo de radiação das décadas de 1968-1977 (C) e de 1978-1987 (D) no Nordeste do Brasil 


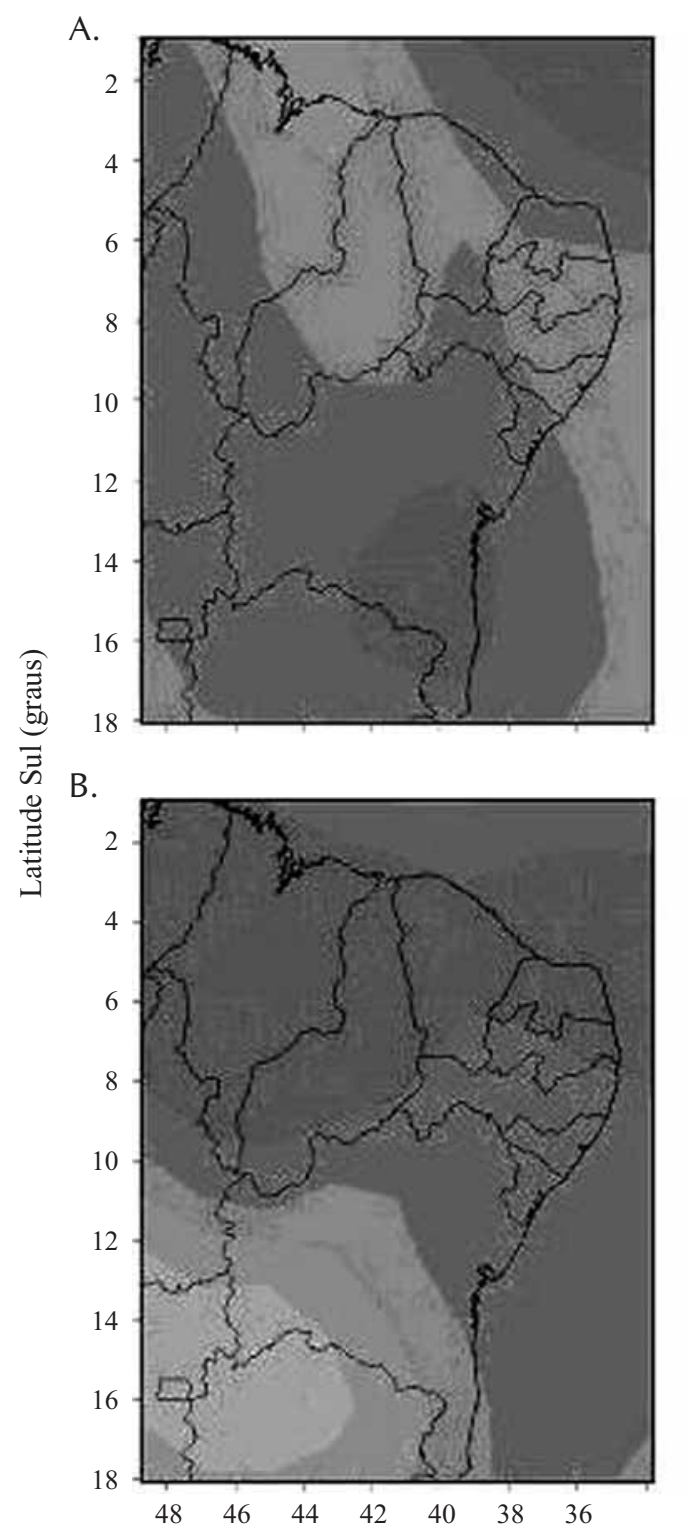

4,0

D

D.

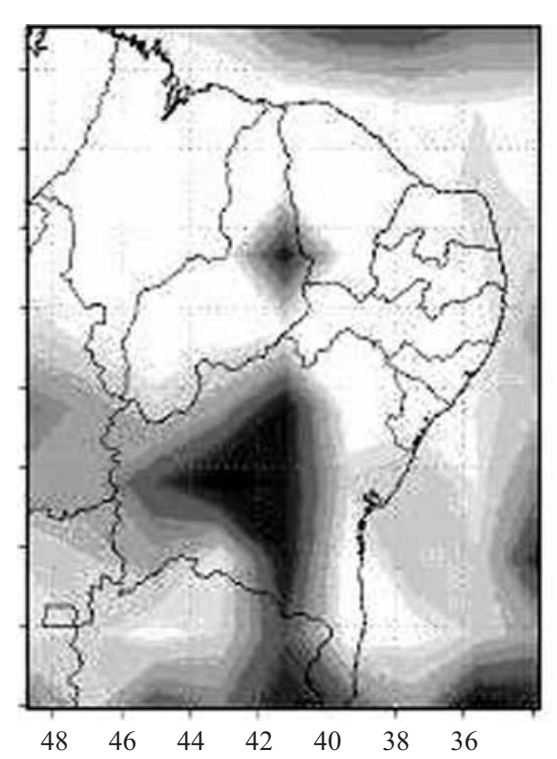

100

80

60

40

Longitude Oeste (graus)

Figura 4. Distribuição espacial da tendência do saldo de radiação das décadas de 1988-1997 (A) e de 1998-2006 (B) e distribuição espacial dos valores dos níveis de significância das tendências do saldo de radiação das décadas de 1988-1997 (C) e de 1998-2006 (D) no Nordeste do Brasil

cia no Nordeste do Brasil, referente ao período total de estudo, é apresentada na Figura 5. Observam-se tendências negativas a oeste do Nordeste do Brasil e positivas no leste da região (Figura 5A). Na parte sudeste da região Nordeste do Brasil são encontrados núcleos de tendências negativas estatisticamente significativos a níveis de 1 a 5\% de probabilidade enquanto a parte central da região não apresentou tendência estatisticamente significativa pelo teste de MannKendall (Figura 5B).

Os resultados desse estudo revelam que, quando a análise é por década, há uma variabilidade na inclinação das tendências ao longo do período de estudo, predominando tendências negativas até a década de 1978-1987 e uma inversão, ou seja, tendências positivas, até a década de 19982006. Várias pesquisas indicam que esta variabilidade da radiação solar, observada até a quarta década, ocorreu em consequência das altas taxas de aerossóis antropogênico, assim como em face das mudanças na cobertura do solo e maior frequência de nebulosidade. No estudo sobre a região Nordeste do Brasil sugere-se que o comportamento decrescente da radiação solar, entre 1948 e 1987, tem relação com a maior frequência de eventos de La Niña de intensidade forte na região. De acordo com as informações do CPC (2008) neste período ocorreram nove eventos de La Niña de intensidade forte na região Nordeste do Brasil; sabe-se, contudo, que os eventos de La Niña e El Niño estão associados a chuvas acima e abaixo do normal, respectivamente, no Nordeste Brasileiro, e podem influenciar na frequência de nebulosidade. Fedorova \& Carvalho (2006) argumentam que em anos de La Niña as regiões equatoriais frequentemente apresentam nebulosidade em 2/3 da latitude ou em toda ela, enquanto em ano de El Niño a frequência de nebulosidade é menor.

O comportamento particular do saldo de radiação nas 


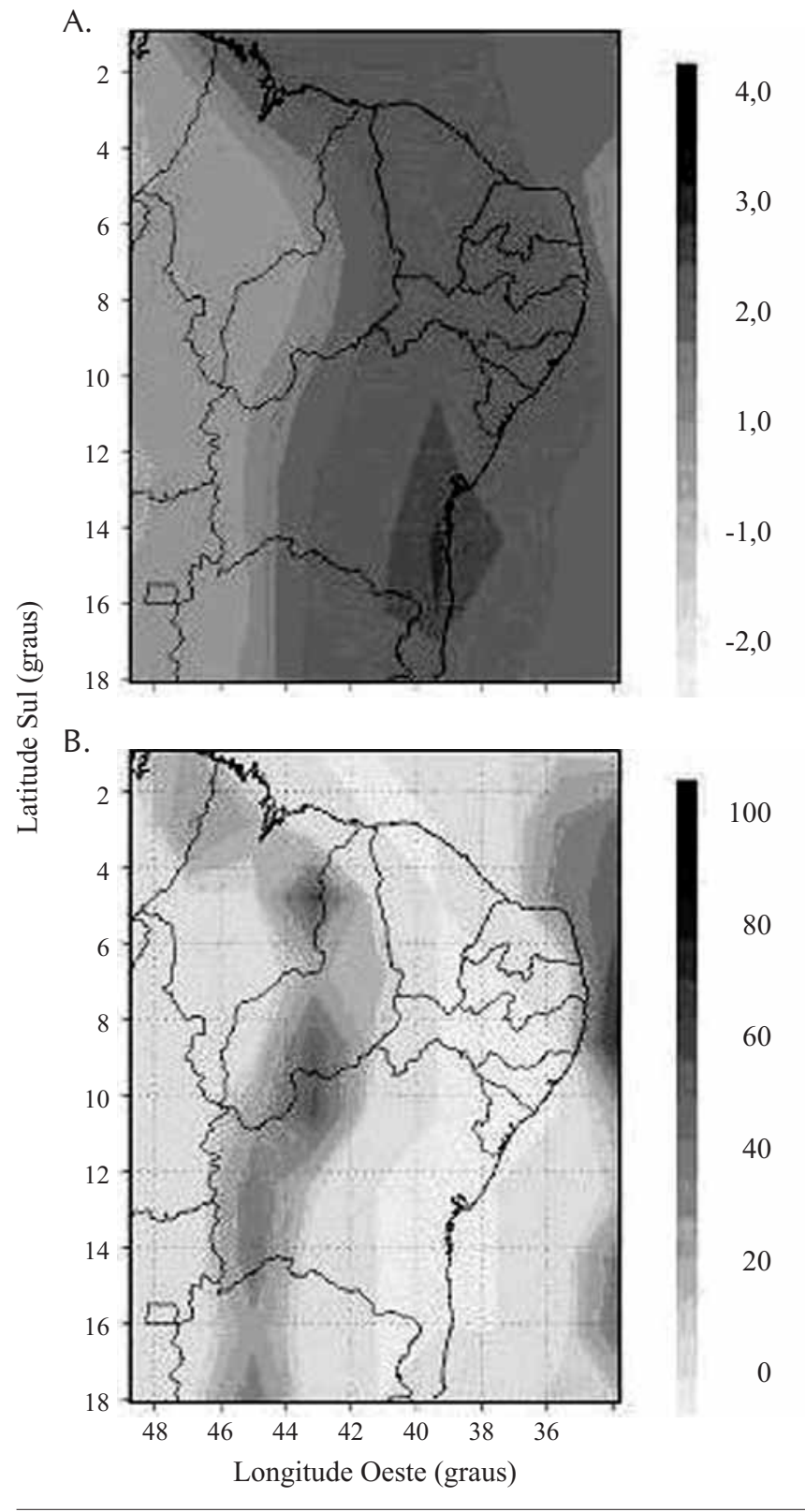

Figura 5. Distribuição espacial da tendência temporal do saldo de radiação (A) e nível de significância do saldo de radiação (B) no N ordeste do Brasil para o período total de estudo (1948-2006)

quatro primeiras décadas no Nordeste do Brasil, pode ter sido influenciado pela maior frequência de fenômenos atmosféricos que intensificam a nebulosidade sobre esta região, haja vista que as nuvens atuam como elemento absorvedor e espalhador da radiação solar (Liang \& Xia, 2005); além disso, os autores afirmam que a cobertura de nuvens pode contribuir, pelo menos em parte, para o declínio da radiação solar observada em algumas regiões do globo. Lohmann et al. (2006) destacam que as concentrações de aerossóis, provenientes das grandes erupções vulcânicas, são responsáveis, em parte, pelo decréscimo nas taxas de radiação solar observadas em suas pesquisas; desta forma, as erupções ocorridas em 1963, 1982 e 1991, respectivamente, no Monte Agung na Indonésia, Chichón no México e Pinatubo, nas Filipinas, também podem ter uma parcela de contribuição nas tendências de radiação solar observadas sobre o NEB. Os trabalhos de Minnis et al. (1993), Molion et al. (2002) e Sant'Anna Neto et al. (2001) reforçam o conceito de que erupções vulcânicas podem reduzir os fluxos de radiação solar sobre a América do Sul e, consequentemente, o decréscimo da radiação solar incidente (ondas curtas) e de saldo de radiação solar.

A predominância de tendências positivas de radiação solar a partir da década de 90, pode ser justificada pela maior frequência de eventos de El Niño, assim como pela fase positiva (quente) da Oscilação Decadal do Pacífico (ODP). De acordo com informações do CPC (2008), entre 1988 e 2006 ocorreram cinco eventos de El Niño de intensidade forte e apenas três eventos La Niña, também de intensidade forte. Segundo Christy \& Spencer (2004) as temperaturas médias globais aumentam quando da ocorrência de El Niño e diminuem quando da ocorrência de La Niña o que, consequentemente, pode provocar alteração do balanço radiativo da Terra. A maior frequência de eventos El Niño também pode ter influenciado no comportamento médio da radiação solar, diminuindo a quantidade de vapor d'água sobre o NEB, haja vista que menos vapor d’água na atmosfera torna mais transparente a radiação solar. Para Cavalcanti (2001) o transporte de umidade para o interior do Nordeste do Brasil é feito através dos ventos alísios, que transportam ar úmido do Oceano Atlântico Tropical para o interior da região.

A análise para todo o período de estudo apresentou tendências negativas de radiação solar a leste e positivas a oeste do NEB estatisticamente significativas a níveis de 1 e 5\% de probabilidade. Sugere-se que essas tendências estejam correlacionadas com a variação dos sistemas atmosféricos de grande escala atuantes sobre a região, que definem os regimes de precipitação pluvial sobre boa parte dessa área. Os eventos como El Niño/Oscilação Sul (ENOS) e ODP, como mencionado na análise por década, podem ter atuado na intensidade da nebulosidade na região Nordeste do Brasil e, consequentemente, na quantidade de vapor d’água presente na atmosfera da região.

\section{Análise de dados observados à superfície}

As tendências dos dados de radiação solar global e da evaporação do tanque classe "A” observados nas localidades de Petrolina, PE, e Juazeiro, BA, no período de 1975 a 2006, são apresentadas na Figura 6. A radiação solar global e a evaporação do tanque classe "A” das duas localidades apresentaram tendências negativas durante todo o período de estudo.

As tendências da radiação solar global nas duas localidades foram estatisticamente significativas a nível de 5\% de probabilidade (Figuras 6A) enquanto a evaporação do tanque classe "A" da localidade de Juazeiro apresentou tendência estatisticamente significativa a nível de $1 \%$, e de Petrolina a nível de 5\% de probabilidade (Figura 6B). As tendências de radiação solar global e da evaporação do tanque classe " $A$ " em Petrolina foram de $-1,19 \mathrm{~W} \mathrm{~m}^{-2}$ e $-0,01 \mathrm{~mm}$, respectivamente.

Esses valores correspondem a uma redução de 36,89 $\mathrm{W} \mathrm{m}^{-2}$ na radiação global e de $0,31 \mathrm{~mm}$ na evaporação do tanque classe "A" dentro do período analisado. Por outro lado, em 


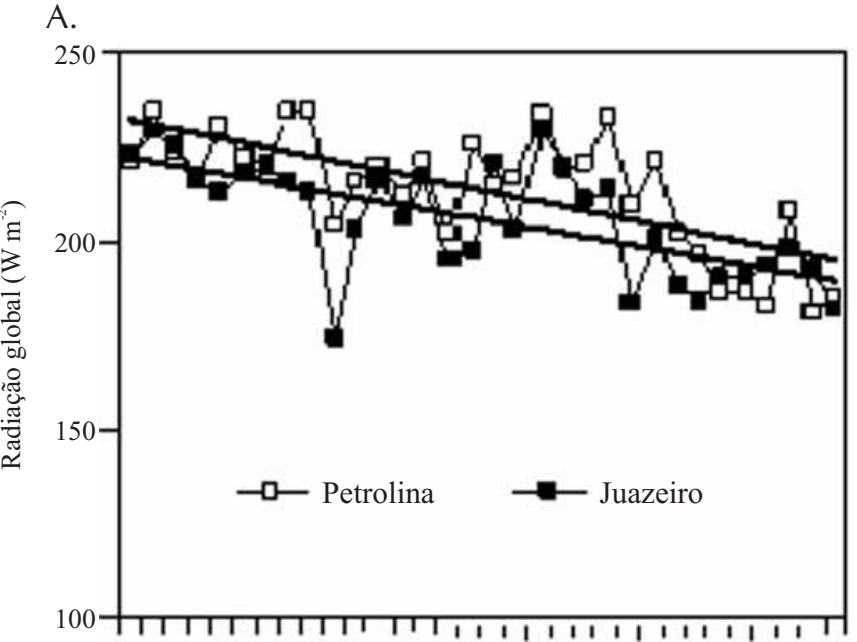

B.

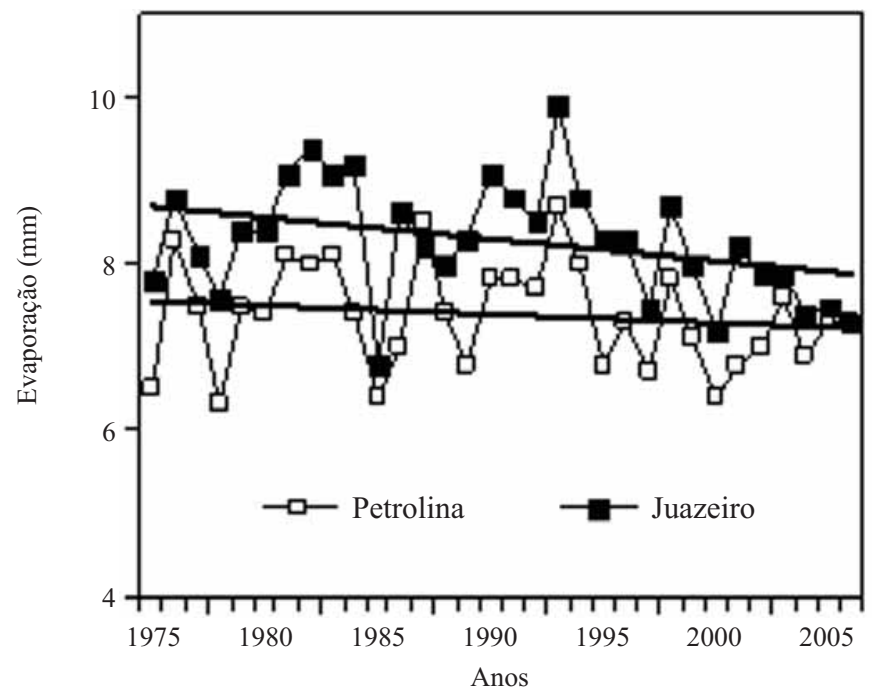

Figura 6. Tendência temporal da radiação solar global (A), evaporação do tanque classe "A" (B) em Petrolina, $P E$, e Juazeiro, BA, para o período de 1975 a 2006

Juazeiro a radiação solar global apresentou tendência de $-1,04 \mathrm{~W} \mathrm{~m}^{-2}$ por ano e a evaporação de $-0,025 \mathrm{~mm}$ por ano, implicando que, no período entre 1975 e 2006, houve redução na radiação global, de $33,28 \mathrm{~W} \mathrm{~m}^{-2}$ e de $0,96 \mathrm{~mm}$ na evaporação do tanque classe “A”. A localidade de Petrolina, PE, apresentou os maiores valores de radiação global e os menores valores de evaporação do tanque classe "A" sendo previsto, entretanto, comportamento inverso, haja vista que, quanto maior a energia disponível maior também é a taxa evaporativa de uma região. Sugere-se que tal situação esteja relacionada à resposta da alteração no albedo da superfície, com expansão do perímetro irrigado na região Senador Nilo Coelho e a construção da barragem de Sobradinho, BA.

As tendências decrescentes dos dados observados de radiação solar global e da evaporação do tanque classe "A", e os dados de saldo de radiação do NCEP/NCAR, indicam a presença do fenômeno "global dimming” na região Nordeste do Brasil. Peterson et al. (1995) e Cohen et al. (2002) afirmaram haver uma relação direta entre a redução na taxa evaporativa e o decréscimo da radiação solar. Esses autores argumentaram que os aerossóis antropogênicos, provenientes de queimadas e de outras alterações no albedo da superfície, também podem ter contribuído na formação do fenômeno "Global dimming”.

\section{CONCLUSÕES}

1. Existem reduções acentuadas no saldo de radiação no Nordeste do Brasil no período entre 1948 a 1987; entretanto, no período entre 1988 a 2006 verificou-se comportamento inverso, com tendências positivas estatisticamente significativas.

2. O período total de estudo (1948 a 2006) exibiu tendências negativas do saldo de radiação estatisticamente significativas a níveis de 1 e 5\% de probabilidade, principalmente na parte leste do NEB.

3. As tendências negativas estatisticamente significativas dos dados observados de radiação solar global e evaporação do tanque classe "A" e do saldo de radiação do NCEP/NCAR estão associadas às alterações no albedo da superfície. Os dados analisados oferecem evidências consistentes do efeito “Global dimming” no Nordeste do Brasil.

\section{LITERATURA CITADA}

Abakumova, G. M. Evaluation of long-term changes in radiation, cloudiness, and surface temperature on territory of the former Soviet Union. Journal Climate, v.9, n.6, p.1319-1327, 1996.

Cavalcanti, E. P. Teor e transporte de vapor d'água na atmosfera do Nordeste do Brasil. Campina Grande: UFPB, 2001. 155p. Tese Doutorado

Ceballos, J. C.; Bottino, M. J. Modelo de estimativa de radiação solar por satélite no CPTEC: Versão GL 1.2. In: Congresso Brasileiro de Meteorologia, 12, 2002, Foz do Iguaçu. Anais... Foz do Iguaçu: SBMET, 2002. CD-Rom

Ceballos, J. C.; Bottino, M. J. Estimativa de radiação solar por satélite: Desempenho do modelo operacional GL1.2. In: Congresso Brasileiro de Meteorologia, 13, 2004, Fortaleza. Anais... Fortaleza: SBMET, 2004, CD-Rom.

Charlson, R. J.; Valero, F. P. J.; Seinfeld, J. H. In search of balance. Science, v.308, p.806-807, 2005.

Christy, J.; Spencer, R. MSU globally averaged atmospheric temperature, global warming debate continues. http://www.ghcc. msfc. nasa. gov/MSU/msusci.html. 16 Dez. 2004.

Cohen, S.; Ianetz, A.; Stanhill, G. Evaporative climate changes at Bet Dagan, Israel, 1964-1998. Agricultural and Forest Meteorology, v.111, n.2, p.83-91, 2002.

CPC - Climate Prediction Center http://www.cpc.ncep.noaa.gov/ products/analysis_monitoring/ensostuff/ensoyears.shtmlmai. 23 Jun. 2009.

Fedorova, N.; Carvalho, M. H. Processos sinóticos em anos de La Niña e de El Niño: Nebulosidade convectiva nas regiões equatoriais e tropicais da América do Sul e oceanos adjacentes. Revista Brasileira de Meteorologia, v.21, n.1, p.1-14, 2006.

Kendall, M. G. Rank correlation measures. London: Charles Griffin, 1975. 220p. 
Liang, F.; Xia X. A. Long-term trends in solar radiation and the associated climatic factors over China for 1961-2000. Annales Geophysicae, v.23, n.7, p.2425-2432, 2005.

Liepert, G. G. Solar radiation in Germany: Observed trends and an assessment of their causes, Part 1. regional approach. Atmospheric Physics, v.67, n.1, p.15-29, 1994.

Liepert B. G. Regionale klimadiagnose mittels messungen der solar en strahlung. Munich: University of Munich, 1996. 176p. Ph.D. Thesis

Liepert, B. G.; Tegen, I. Multi-decadal solar radiation trends in the United States and Germany and direct tropospheric aerosol forcing. Journal of Geophysical Research, v.107, n.D12, 2002.

Lohmann, S.; Schillings, C.; Mayer, B.; Meyer, R. Long-term variability of solar direct and global radiation derived from ISC$\mathrm{CP}$ data and comparison with reanalysis data. Solar Energy, v.80, n.11, p.1390-1401, 2006.

Mann, H. B. Econometrica. The Econometric Society, v.13, n.3, p.245-259, 1945.

Minnis, P.; Harison, E. F.; Stowe, L. L.; Gibson, G. G.; Denn, F. M.; Doelling, D. R.; Smith Júnior, W. L. Radiative climate forcing by Mount Pinatubo eruption. Science, v.259, p.1411-1415, 1993.

Modares, R.; Silva, V. de P. R. de. Trends analysis of rainfall record in arid and semi arid regions of Iran. Journal of Arid Environments, v.70, n.1, p.344-355, 2007.

Molion, L .C. B.; Querino, C. A. S.; Menezes, D. C.; Lima, E. A.; Silva, H. O.; Borba, J. C. C. Possíveis efeitos de aerossóis vulcânicos no balanço radiativo da reserva biológica nacional do Jarú na Amazônia. In: Congresso Brasileiro de Meteorologia, 12, 2002, Foz do Iguaçu. Anais... Foz do Iguaçu: SBMET, 2002. CD-Rom

Nazarenko, L.; Menon, S. Varying trends in surface energy fluxes and associated climate between 1960-2002 based on transient climate simulations. Journal of Geophysical Research, v.32, L22704, doi:10.1029/2005GL024089, 2005.

Ohmura, A.; Lang, H. Secular variation of global radiation in Europe. In: Lenoble, J.; Geleyn, J. F. (ed.): IRS’88: Current problems in atmospheric radiation. Deepak Publishing, v.1, p.298-301, 1989.

Pallé, E.; Goode, P. R.; Montanés-Rodrýguez P.; Koonin S. E. Changes in earth's reflectance over the past two decades. Science, v.304, n.5675, p.1299-1301, 2004.

Peterson, T. C.; Golubev, V. S.; Groisman, P. Y. Evaporation losing its strength. Nature, v. 377, n.6551, p.687-688, 1995.

Philipona, R.; Durr, B.; Marty, C.; Ohmura, A.; Wild, M. Radiative forcing-measured at Earth's surface - corroborate the increasing greenhouse effect. Geophysical Research Letters, v.31, p.L03202, 2004.

Qian, Y.; Kaiser, D. P.; Leung, L. R.; Xu, M. More frequent cloudfree sky and less surface solar radiation in China from 1955 to 2000. Geophysical Research Letters, v.1, n.33, L01812, 2006.

Roderick, M. L.; Farquhar, G. D. Changes in Australian pan evaporation from 1970 to 2002. International Journal of Climatology, v.24, p.1077-1090, 2004.
Russak, V. Trends of solar radiation, cloudiness and atmospheric transparency during recent decades in Estonia. Tellus, v.42B, p.206-210, 1990.

Sant'Anna Neto, J. L.; Yamabe, T. H.; Tommaselli, J. T. G. A influência da atividade vulcânica na variabilidade das temperaturas médias mensais do Estado de São Paulo. In: Simpósio Brasileiro de Geografia Física Aplicada, 9, 2001, Recife. Anais... Recife: UPE, 2001. CD-Rom.

Silva, R. A.; Santos, D. N.; Silva, V. de P. R. da ; Cavalcanti, E. P. Tendência da radiação solar no nordeste do Brasil com base nos dados do NCEP/NCAR. In: Congresso Brasileiro de Meteorologia, 15, 2008, São Paulo. Anais... São Paulo: SBMET, 2008, CD-Rom.

Silva, V. de P. R. da. On climate variability in Northeast of Brazil. Journal of Arid Environments, v.58, p.575-596, 2004.

Silva, V. de P. R. da; Cavalcanti, E. P.; Braga, C. C.; Azevedo, P. $\mathrm{V}$. Evaluating trends in solar radiation based on data fields from the NCEP/NACR reanalysis and measurements, 7th EMS Annual Meeting/8th ECAM. El Escorial, 2007, Madrid Anais... Madrid: European Meteorogical Society, 2007. CD-Rom.

Silva, V. de P. R. da; Sousa, F. A. S.; Cavalcanti, E. P.; Souza, E. P.; Silva, B. B. da. Teleconnections between sea-surface temperature anomalies and air temperature in northeast Brazil. Journal of Atmospheric and Solar-Terrestrial Physics, v.68, n.1, p.781-792, 2006.

Stanhill, G.; Cohen, S. Recent changes in solar irradiance in Antarctica. Journal of Climate. v.10, n.8, p.2078-2086, 1997.

Stanhill, G.; Cohen, S. Global dimming: A review of the evidence for a widespread and significant reduction in global radiation with discussion of its probable causes and possible agricultural consequences. Agricultural and Forest Meteorology, v.107, n.1, p.255-278, 2001.

Stanhill, G.; Ianetz, A. Long term trends in, and spatial variation of global irradiance in Israel, Tellus, v.49B, n.1, p.112-122, 1996.

Stanhill, G.; Kalma, J. D. Secular variation of global irradiance in Australia. Australian Meteorological Magazine, v.43, p.81-86, 1994.

Stanhill, G.; Kalma, J. D. Solar dimming and urban heating in Hong Kong. International Journal of Climatology, v.15, n.1, p.933-941, 1995.

Tebakari, T.; Yoshitani, J.; Suvanpimol, C. Time-space trend in pan-evaporation over Thailand. Journal of Hydrologic Engineering, v.10, n.3, p.205-215, 2005.

Thomas, A. Spatial and temporal characteristics of potential evapotranspiration trends over China. International Journal of Climatology, v.20, p.381-396, 2000.

Yue, P. S.; Yang, T. C.; Wu, C. K. Impact of climate change on water resources in southern Taiwan. Journal of Hydrology, v.260, p.161-175, 2002.

Zhitorchuk, Y. V.; Stadnyuk, V. V.; Shanina, I. N. Study of linear trends of temporal series of solar radiation. Izvestiâ Akademii nauk SSSR, v.30, p.389-396, 1994. 